\title{
Manipulating Localized Molecular Orbitals by Single-Atom Contacts
}

\author{
Weihua Wang, ${ }^{1}$ Xingqiang Shi, ${ }^{2}$ Chensheng Lin, ${ }^{2}$ Rui Qin Zhang, ${ }^{2}$ Christian Minot, ${ }^{2,3}$ Michel A. Van Hove, ${ }^{2}$ \\ Yuning Hong, ${ }^{4}$ Ben Zhong Tang, ${ }^{4}$ and Nian Lin ${ }^{1, *}$ \\ ${ }^{1}$ Department of Physics, The Hong Kong University of Science and Technology, Hong Kong, China \\ ${ }^{2}$ Department of Physics and Materials Science, City University of Hong Kong, Hong Kong, China \\ ${ }^{3}$ Laboratoire de Chimie Théorique, Université Pierre \& Marie Curie Paris 6, \\ CNRS, UMR7616, Case 137, 4 place Jussieu, Paris, F-75252 Cedex France \\ ${ }^{4}$ Department of Chemistry, The Hong Kong University of Science and Technology, Hong Kong, China
}

(Received 31 May 2010; published 13 September 2010)

\begin{abstract}
We have fabricated atom-molecule contacts by attachment of single $\mathrm{Cu}$ atoms to terpyridine side groups of bis-terpyridine tetra-phenyl ethylene molecules on a $\mathrm{Cu}(111)$ surface. By means of scanning tunneling microscopy, spectroscopy, and density functional calculations, we have found that, due to the localization characteristics of molecular orbitals, the $\mathrm{Cu}$-atom contact modifies the state localized at the terpyridine side group which is in contact with the $\mathrm{Cu}$ atom but does not affect the states localized at other parts of the molecule. These results illustrate the contact effects at individual orbitals and offer possibilities to manipulate orbital alignments within molecules.
\end{abstract}

DOI: 10.1103/PhysRevLett.105.126801

PACS numbers: 73.20.Hb, 68.37.Ef, 73.63.Rt, 85.65.+h

Metal-molecule contacts play a decisive role in molecule-based electronic devices [1,2]. The contacts may dominate the single-molecule conductance through defining the molecule-metal coupling strength as well as modifying the alignment of molecular orbitals (MOs) within the molecule and/or with respect to the contact electrode states [3]. Thus it is of great importance to obtain insight into the molecular electronic characteristics with atom-molecule contacts. In recent years, this issue has been addressed by various techniques, including scanning probe microscopies, break junctions, electromigration, as well as theoretical modeling [4-13]. Among these, scanning tunneling microscopy (STM) provides valuable information on how a particular contact modifies the molecular orbitals owing to STM's capability to reveal the geometrical details of the contacts at the single-molecule level, while at the same time measuring electronic properties by means of scanning tunneling spectroscopy (STS). Recently STM has been used to measure single-molecule conductance owing to its capability to manipulate or contact single molecules [14-16].

Various reports demonstrate that MOs are shifted by the attachment of a single atom or atom clusters [17-23]. So far, most of these studies addressed relatively small molecules. Here we report on an investigation of single-atom contacts with a complex molecule consisting of two side functional groups linked by a bridging group, which represents a prototype molecular wire. Our results demonstrate that attachment of a single metal atom to a molecular side group shifts the energy levels of the MOs that are in direct contact with the metal atom without affecting the MOs localized at other parts. Taking advantage of this localized modification of MOs, we were able to realign the MO energy levels within a molecule.
The molecule used in this study is bis-terpyridine tetraphenyl ethylene (BTP-TPE, $\sim 2.5 \mathrm{~nm}$ long), as shown in Fig. 1(a). This molecule has two terpyridine (tp) side groups that are linked symmetrically to a central tetraphenyl ethylene (tpe) group through phenyl (ph) groups. This molecule possesses several highly localized MOs: two degenerate low-lying unoccupied MOs (LUMO +2 and
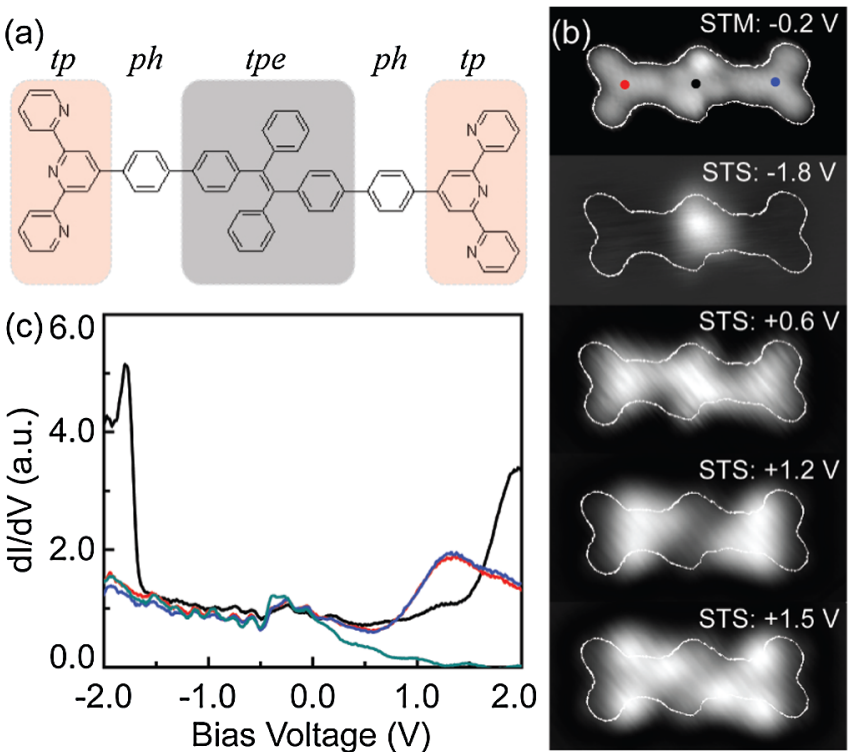

FIG. 1 (color online). (a) Molecular structure of BTP-TPE. (b) STM image and STS maps acquired at the indicated bias voltages. The molecular contour of the STM topograph (white line) is overlaid on the STS maps. Image size: $5.0 \times 2.5 \mathrm{~nm}^{2}$. (c) STS spectra acquired at three specific sites marked in the top panel in (b) following the color code. The cyan spectrum was acquired at the $\mathrm{Cu}$ surface. 
LUMO + 3) are localized at the two tp side groups while the highest occupied MO (HOMO) is localized at the central tpe group. By controlling the sample preparation conditions, $\mathrm{Cu}$ adatoms available on the surface may spontaneously coordinate to tp side groups. When asymmetric coordination occurs, i.e., only one tp side group is attached to a $\mathrm{Cu}$ adatom, we find that the orbital localized at the uncoordinated $(\mathrm{Cu}$-free) tp side group is unaffected, while the orbital localized at the $\mathrm{Cu}$-coordinated tp side group is down-shifted in energy. So the orbital degeneracy of LUMO +2 and LUMO + 3 is lifted. In a symmetric coordination where both tp side groups are attached to $\mathrm{Cu}$ atoms, the MOs localized at both side groups show the same down-shifted energies, so the orbital degeneracy is preserved. We also found that the HOMO is not affected by either symmetric or asymmetric coordination.

The experiments were performed in a low-temperature ultrahigh vacuum scanning tunneling spectroscope system (Omicron) operated at $4.9 \mathrm{~K}$. BTP-TPE molecules were evaporated from an organic molecular evaporator at $405 \mathrm{~K}-425 \mathrm{~K}$ and deposited on the $\mathrm{Cu}(111)$ surface. To adsorb intact molecules, the substrate was cooled down to $180 \mathrm{~K}$ during molecular deposition. Density-functional theory (DFT) calculations were performed within the projector-augmented wave (PAW) plane-wave approach as implemented in the VASP code [24,25]. The exchangecorrelation functional was treated with the generalized gradient approximation in the Perdew-Burke-Ernzerhof (PBE) form [26]. The STM images were simulated in constant current mode with the Tersoff-Hamann approximation (i.e., computing an isodensity surface $z(x, y)$ above the adsorbed molecule) [27,28].

A representative STM topograph of a single molecule is shown in Fig. 1(b) (top panel), clearly resolving the central tpe group and the two tp side groups, which exhibits the $C_{2}$ symmetry of the molecule. Figure 1 (c) shows $d I / d V$ tunneling spectra acquired at three specific locations within the molecule [marked by the black and colored dots in Fig. 1(b)]. The spectra taken near the two sides exhibit similar features: a broad peak at $1.3 \mathrm{~V}$. The spectrum taken at the central position shows a peak at $-1.8 \mathrm{~V}$ and a rise above $1.5 \mathrm{~V}$. The other panels in Fig. 1(b) show STS maps at the indicated bias voltages, which illustrate the spatial distribution of the specific states. These maps indicate that the state at $-1.8 \mathrm{~V}$ is mostly localized at the central tpe group, the state at $1.2 \mathrm{~V}$ is mostly distributed at the two sides in $C_{2}$ symmetry, the state at $1.5 \mathrm{~V}$ is distributed at the flanks of the tp groups as well as at ph groups, and the state at $0.6 \mathrm{~V}$ is delocalized. The site-dependent tunneling spectra as well as the STS maps confirm that the molecular states at $-1.8 \mathrm{~V}, 1.2 \mathrm{~V}$ and $1.5 \mathrm{~V}$ are highly localized.

Figure 2(a) shows the DFT optimized adsorption structure of a BTP-TPE molecule on a single $\mathrm{Cu}$ layer (detailed analysis has verified that the results are insensitive to the number of $\mathrm{Cu}$ layers, four or one, used in the calculations [28]). The simulated STM image of the adsorbed molecule is shown in the top panel of Fig. 2(b), which reproduces

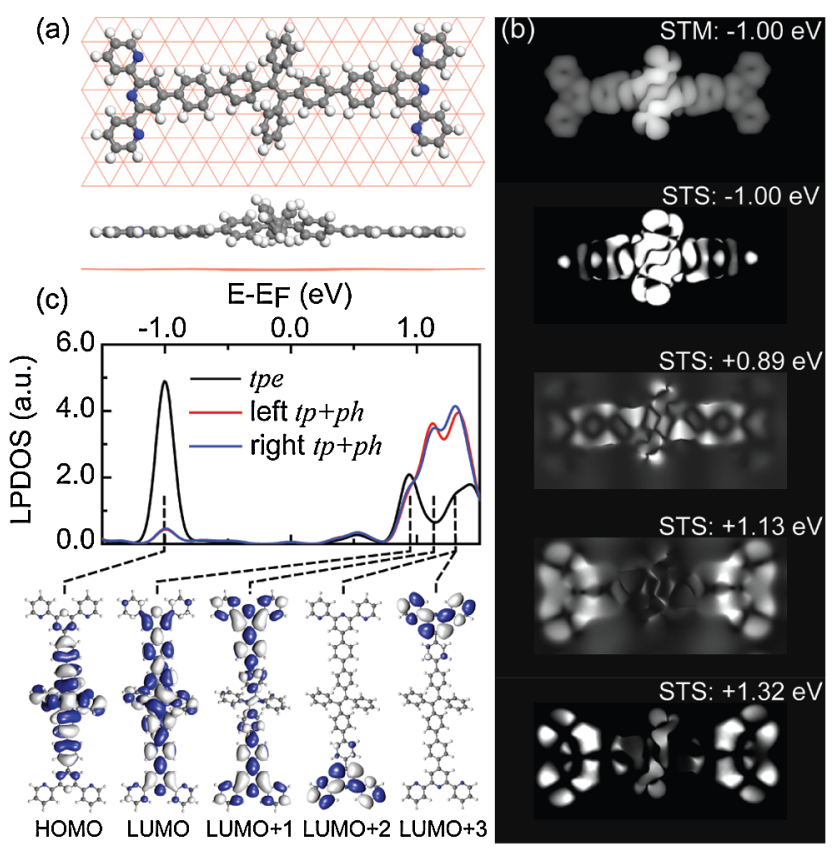

FIG. 2 (color online). (a) Top and side views of a DFT optimized molecule adsorbed on one layer of $\mathrm{Cu}$ atoms. (b) Simulated STM image and PDOS maps at the indicated energies. Image size: $5.0 \times 2.5 \mathrm{~nm}^{2}$. (c) Integrated LPDOSs of the two side groups and the central group. The frontier MOs of a free molecule are shown below the LPDOS.

the main features of the experimental STM topograph. To simulate the site-dependent tunneling spectra, we separately integrated the projected density of states of three groups-one for the central tpe part and two for the side parts, each including a tp and a ph group-denoted as local projected density of states (LPDOSs), as shown in Fig. 2(c) (the Fermi level is set midway between HOMO and LUMO). The two side groups possess very similar LPDOSs, showing a double-peak feature at $1.1 / 1.3 \mathrm{eV}$. The central tpe group shows a strong peak at $-1.0 \mathrm{eV}$, a very weak peak at $0.5 \mathrm{eV}$ and two moderate peaks at $0.9 \mathrm{eV}$ and $1.4 \mathrm{eV}$. In Fig. 2(c) we plot the frontier MOs of a free BTP-TPE molecule. One can see that the HOMO is mostly localized at the central tpe, while the LUMO +2 and LUMO +3 are mostly localized at the tp groups. (Note that LUMO +2 and LUMO +3 are two degenerate MOs localized at opposite tp groups.) The calculation allows us to assign the LPDOS peaks to the frontier MOs of the free molecule, as indicated by the dashed lines. (Note: due to the common DFT underestimation of gap values, the calculated LPDOS peak positions are closer to the Fermi level in comparison with the measured values.) The other panels in Fig. 2(b) present simulated maps of PDOS at the indicated energies. Consistent with the LPDOSs shown in Fig. 2(c), the state at $-1.0 \mathrm{eV}$ is mostly located at the molecular center, while the states at $1.1 \mathrm{eV}$ and $1.3 \mathrm{eV}$ are mostly located at the two sides. These theoretical results are in fair agreement with the experimental results shown 
in Fig. 1 and support the experimental conclusion that these MOs are highly localized.

In the next step, we used $\mathrm{Cu}$ adatoms to form single-atom contacts with the tp side groups [29,30]. Experimentally, we deposited the molecules onto a substrate held at room temperature with a lower deposition rate. This procedure can effectively enhance the probability of the coordination of $\mathrm{Cu}$ adatoms with the terpyridine functions [31,32]. We have identified many molecules showing asymmetric characteristics on these samples. Figure 3(a) (top panel) shows a typical STM topograph of such a molecule acquired at $-1.8 \mathrm{~V}$ bias voltage. At first sight, it does not exhibit an obvious asymmetric appearance. This can be understood through DFT calculations. The DFT optimized structure of an asymmetrically coordinated molecule, whose right-side tp side group is coordinated to a $\mathrm{Cu}$ atom, is shown in Fig. 3(c). The $\mathrm{Cu}$ atom is lower than the tp side groups by about $1.6 \AA$. Figure $3(\mathrm{~b})$ (top panel) shows a simulated $(-1.0 \mathrm{eV})$ STM image of the asymmetrically coordinated molecule. One may note that the two sides show no obvious differences in the simulated image, consistent with the STM topograph.

The coordination-induced asymmetry is clearly resolved in STS data. Figure 3(d) shows site-dependent tunneling spectra. The spectra acquired at the left side and at the center exhibit the same features as those of the uncoordinated molecule shown in Fig. 1(c), while the spectrum acquired at the right side shows a peak at $0.9 \mathrm{~V}$, which is $0.4 \mathrm{~V}$ down-shifted compared with the $1.3 \mathrm{~V}$ peak in the spectrum of the left side. The other panels in Fig. 3(a) show STS spatial maps of the asymmetric molecule at the same energies as those shown in Fig. 1(b). One may see that the unoccupied states at $0.6 \mathrm{~V}, 1.2 \mathrm{~V}$, and $1.5 \mathrm{~V}$, which are
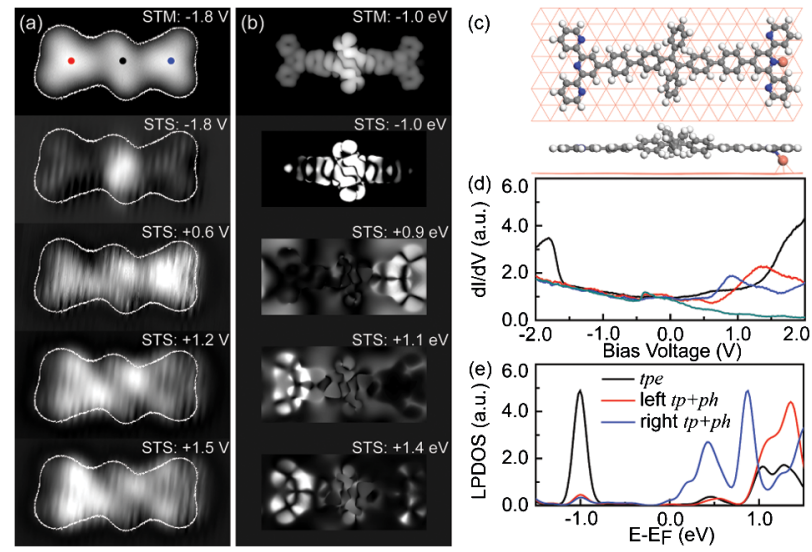

FIG. 3 (color online). (a) STM image and STS maps acquired at the indicated bias voltages of a single-side coordinated BTPTPE. (b) DFT simulated STM image and PDOS maps at the indicated energies. Images in (a) and (b) have the same scale with a size of $5.0 \times 2.5 \mathrm{~nm}^{2}$. (c) Top and side views of a DFT optimized single-side coordinated molecule adsorbed on one layer of $\mathrm{Cu}$ atoms. (d) STS spectra acquired at three specific sites as marked in the topmost panel in (c). The cyan spectrum was acquired at the $\mathrm{Cu}$ surface. (e) Integrated LPDOSs of the two side groups and the central group. symmetric in the former case, become asymmetric. In particular, the right group shows a stronger signal at $0.6 \mathrm{~V}$ and weaker signals at $1.2 \mathrm{~V}$ and $1.5 \mathrm{~V}$.

The calculated electronic structures of the asymmetrically coordinated molecule are shown in Figs. 3(b) and 3(e). The LPDOSs [in Fig. 3(e)] of the uncoordinated side group and the central tpe group exhibit the same features as those of the uncoordinated molecule as shown in Fig. 2(c). In contrast, the LPDOS of the coordinated side shows two peaks at $0.5 \mathrm{eV}$ and $0.9 \mathrm{eV}$. The $0.9-\mathrm{eV}$ peak can be assigned as down-shifted $1.3 \mathrm{eV}$ peak of the uncoordinated tp. Figure 3(b) presents the simulated PDOS maps of the asymmetrically coordinated molecule. One can see that the $0.9-\mathrm{eV}$ map shows a stronger signal at the coordinated side and the 1.1-eV and 1.4-eV maps show a stronger signal at the uncoordinated side. These theoretical results agree very well with the experimental data, further supporting that the observed symmetry breaking of the low-lying unoccupied states is given by the single-side $\mathrm{Cu}$ coordination. Furthermore, the HOMO, which is localized at the molecule center, remains almost unchanged, indicating that the $\mathrm{Cu}$ coordination does not influence it. This explains the absence of the asymmetric appearance of the $-1.8 \mathrm{~V}$ STM image: The $-1.8 \mathrm{~V}$ image is contributed by the states whose energy falls between the Fermi level and the HOMO. These states are not affected by the $\mathrm{Cu}$ coordination; thus, the STM topograph does not show an asymmetric shape.

These results imply the degenerate LUMO +2 and LUMO +3 of the uncoordinated molecules lose their degeneracy under the asymmetric coordination. The theory provides an explanation of this local modification of the molecular states. When a $\mathrm{Cu}$ atom binds to one tp group, the MO which is localized at this group (LUMO +2 ) is modified. However, LUMO +3 , which is localized at the other tp side group, is not affected because the spatial overlap of this state with the $\mathrm{Cu}$ atom is negligible. Similarly, due to its local nature, the $\mathrm{HOMO}$ is not affected by $\mathrm{Cu}$ coordination either. Hence the localized contact effects can be attributed to the highly localized MO configuration.

A downshift in the energy of the unoccupied states is a quite general observation in molecule-metal contacts [17]. This effect is ascribed to charge transfer or charge redistribution inside the molecule resulting from the interaction with the metal for the empty states. Since the energy downshift observed in the present study occurs locally, we expect that the charge transfer or redistribution is also a local effect. We have carried out a Bader charge analysis on the asymmetrically coordinated molecules and found that the pyridine unit which is coordinated to the $\mathrm{Cu}$ carries a charge of $-0.14|e|$, and the two side pyridine units $+0.06|e|$ each. In the other parts of the molecule, including the intact tp side group and the central tpe group, the charge states are not affected. In particular, the three "pyridine" units of the intact tp group all carry virtually zero net charge.

Now we discuss symmetrically coordinated molecules. Figure 4(a) (top panel) shows a STM topograph of such a 


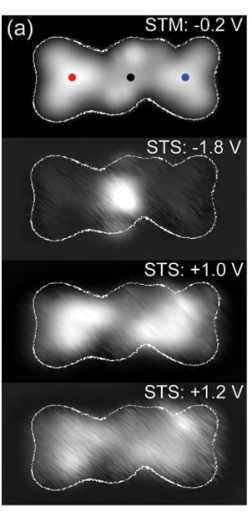

(f)
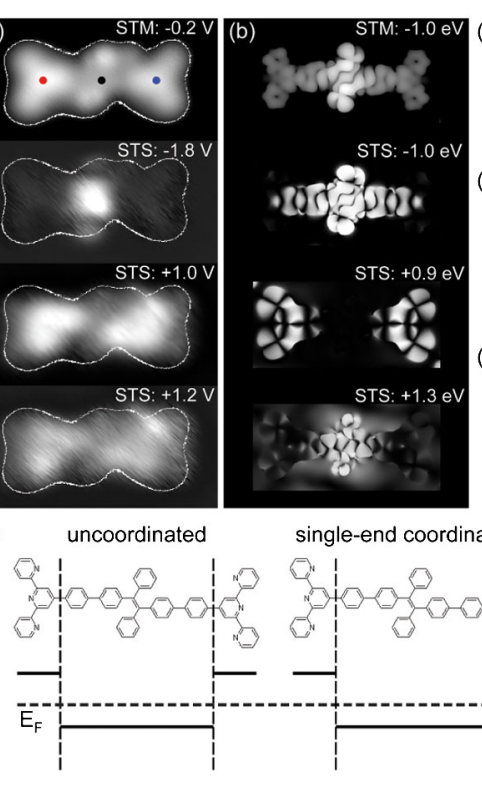

single-end coordinated
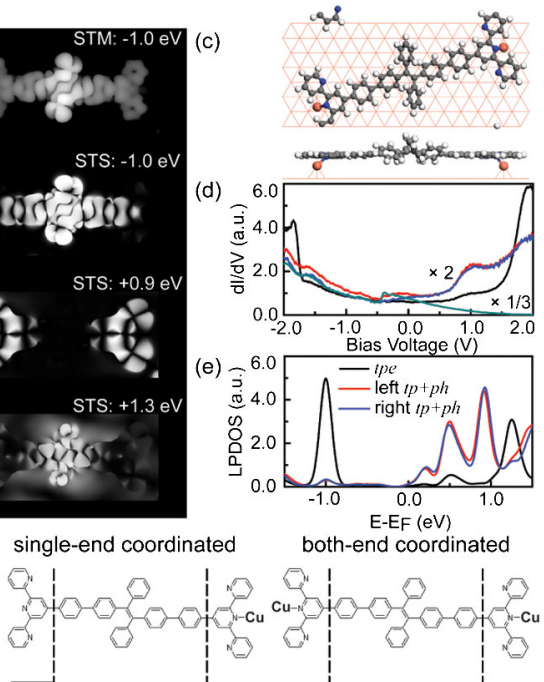

both-end coordinated

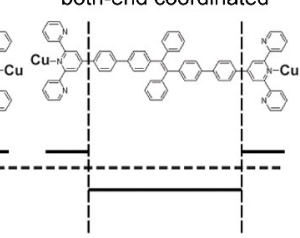

FIG. 4 (color online). (a) STM image and STS maps acquired at the indicated bias voltages of a double-side coordinated BTPTPE. (b) DFT simulated STM image and PDOS maps at the indicated energies. Images in (a) and (b) have the same scale with a size of $5.0 \times 2.5 \mathrm{~nm}^{2}$. (c) Top and side views of a DFT optimized double-side coordinated molecule adsorbed on one layer of $\mathrm{Cu}$ atoms. (d) STS spectra acquired at three specific sites as marked in the topmost panel in (c). The cyan spectrum was acquired at the $\mathrm{Cu}$ surface. (e) Integrated LPDOSs of the two side groups and the central group. (f) Scheme showing the realignment of the localized MOs by single-atom contacts.

molecule. The site-dependent tunneling spectra shown in Fig. 4(d) reveal that the two tp groups exhibit a similar peak at $1.0 \mathrm{~V}$ and a shoulder at $1.7 \mathrm{~V}$, while the central tpe shows the same features as those of the uncoordinated or single-side coordinated molecules. The STS maps shown in Fig. 4(a) reveal that the states at $1.0 \mathrm{~V}$ and $1.2 \mathrm{~V}$ clearly possess a $C_{2}$ symmetric distribution, indicating that the states localized at the two tp groups are modified equally. The DFT optimized structure of the symmetrically coordinated molecule is shown in Fig. 4(c). The LPDOSs [shown in Fig. 4(e)] of both sides posses the same features as the coordinated side group of the previous molecule. The LPDOS of the central tpe group shows a $-1.0 \mathrm{eV}$ peak, which is the same as that of the uncoordinated or the singleside coordinated molecule (with an additional peak at $1.3 \mathrm{eV}$ ). Figure 4(b) presents the simulated STM and LPDOS maps of the symmetrically coordinated molecule. One can see that STS maps at all energies show a symmetric appearance. These results indicate that the orbital degeneracy of LUMO +2 and LUMO +3 is preserved with the symmetrical coordination.

In summary, we have demonstrated that in a complex molecule consisting of two side groups and a bridging group, owing to the localization of specific MOs, these
MOs can be manipulated individually by single-atom contacts. As illustrated in Fig. 4(f), the MOs localized at the two sides can be down-shifted individually through $\mathrm{Cu}$ coordination without affecting the MO localized at the molecular center.

This work was supported by the Grant of HKUST RPC07/08.SC02, Hong Kong RGC Grant No. CityU 102408, and the CityU Centre for Applied Computing and Interactive Media. C. M. is grateful to the French Consulate General in Hong Kong and Macau for financial support.

*To whom all correspondence should be addressed. phnlin@ust.hk

[1] A. Aviram and M. A. Ratner, Chem. Phys. Lett. 29, 277 (1974).

[2] R. L. McCreery, Chem. Mater. 16, 4477 (2004).

[3] N. J. Tao, Nature Nanotech. 1, 173 (2006).

[4] L. A. Bumm et al., Science 271, 1705 (1996).

[5] M. A. Reed et al., Science 278, 252 (1997).

[6] C. Kergueris et al., Phys. Rev. B 59, 12505 (1999).

[7] V. J. Langlais et al., Phys. Rev. Lett. 83, 2809 (1999).

[8] H. Park et al., Nature (London) 407, 57 (2000).

[9] X. D. Cui et al., Science 294, 571 (2001).

[10] J. Park et al., Nature (London) 417, 722 (2002).

[11] J. Reichert et al., Phys. Rev. Lett. 88, 176804 (2002).

[12] H. B. Weber et al., Chem. Phys. 281, 113 (2002).

[13] J. Reichert et al., Appl. Phys. Lett. 82, 4137 (2003).

[14] L. Lafferentz et al., Science 323, 1193 (2009).

[15] D. Kockmann, B. Poelsema, and H. J. W. Zandvliet, Nano Lett. 9, 1147 (2009).

[16] W. Haiss et al., Nature Mater. 5, 995 (2006).

[17] G. V. Nazin, X. H. Qiu, and W. Ho, Science 302, 77 (2003).

[18] R. Yamachika et al., Science 304, 281 (2004).

[19] J. Repp et al., Science 312, 1196 (2006).

[20] D. Wegner et al., Phys. Rev. Lett. 103, 087205 (2009).

[21] Y. J. Song et al., Nano Lett. 10, 996 (2010).

[22] A. Weber-Bargioni et al., J. Phys. Chem. C 112, 3453 (2008).

[23] P. Liljeroth, I. Swart, S. Paavilainen, J. Repp, and G. Meyer, Nano Lett., 10, 2475 (2010).

[24] P. E. Blöchl, Phys. Rev. B 50, 17953 (1994).

[25] G. Kresse and J. Furthmüller, Comput. Mater. Sci. 6, 15 (1996).

[26] J. P. Perdew, K. Burke, and M. Ernzerhof, Phys. Rev. Lett. 77, 3865 (1996).

[27] J. Tersoff and D. R. Hamann, Phys. Rev. B 31, 805 (1985).

[28] X. Shi et al. (to be published).

[29] N. Lin et al., Angew. Chem., Int. Ed. 41, 4779 (2002); R. Ohmann, L. Vitali, and K. Kern, Nano Lett. 10, 2995 (2010).

[30] S. Stepanow, N. Lin, and J. V. Barth, J. Phys. Condens. Matter 20, 184002 (2008).

[31] W. Wang, Y. Hong, X. Shi, B. Z. Tang, C. Minot, M. Van Hove, and N. Lin, J. Phys. Chem. Lett. 1, 2295 (2010).

[32] Molecular manipulation by STM is able to convert the asymmetric molecules to uncoordinated symmetric molecules, thus confirming the asymmetric molecules are due to $\mathrm{Cu}$ coordination of the uncoordinated molecules. 